\title{
Vólvulo del colon transverso espontáneo: reporte de dos casos
}

\section{Two Case Reports of Spontaneous Transverse Colon Volvulus}

Diego Severiche B, MD, ${ }^{1}$ Reinaldo Andrés Rincón S., MD, ${ }^{2}$ Andrés Montoya D., MD, ${ }^{2}$ Alejandro Concha M., MD, ${ }^{2}$

Diana Carolina Hennessey C., MD. ${ }^{3}$

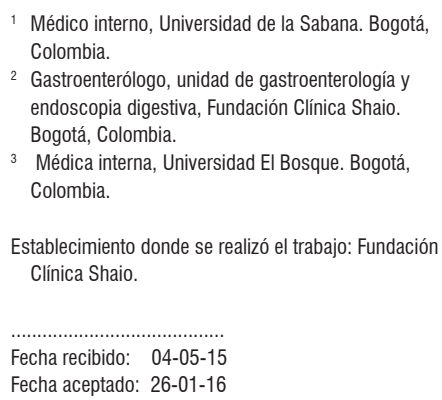

2 Gastroenterólogo, unidad de gastroenterología y endoscopia digestiva, Fundación Clínica Shaio. Bogotá, Colombia.

3 Médica interna, Universidad El Bosque. Bogotá, Colombia.

Establecimiento donde se realizó el trabajo: Fundación Clínica Shaio.

Fecha recibido: $\quad 04-05-15$

Fecha aceptado: 26-01-16

\begin{abstract}
Resumen
Los vólvulos del colon transverso son una presentación poco frecuente del vólvulo del colon debido a la fijación anatómica que impide su torsión. En la literatura mundial se han presentado menos de 100 casos documentados. A continuación se presentan 2 casos de vólvulo del colon transverso que se sospechan mediante cuadro clínico e imagenológico, son llevados a colonoscopia para devolvulación y se confirman en el intraoperatorio.
\end{abstract}

\section{Palabras clave}

Vólvulos, colon transverso, colonoscopia, obstrucción intestinal, dolor abdominal.

\begin{abstract}
Transverse colon volvulus is rare due to the anatomic location which impedes twisting. In the literature of the world, there have been fewer than 100 documented cases. This article presents two cases of transverse colon volvulus. They were suspected because of clinical evidence, images were obtained, colonoscopic reduction was performed and the disorders were confirmed intraoperatively.
\end{abstract}

Keywords

Volvulus, transverse colon, colonoscopy, intestinal obstruction, abdominal pain.

\section{INTRODUCCIÓN}

El término vólvulo se origina del latín volveré, que significa torsión, y se refiere a la torsión del colon sobre su propio eje (principalmente del sigmoide), que puede desencadenar una obstrucción intestinal con desarrollo de isquemia y necrosis, llevando a perforación, peritonitis, sepsis y hasta la muerte $(1,2)$.

El vólvulo representa entre el 10\%-15\% de los casos de obstrucción del colon y entre un $1 \%$ y $20 \%$ de todas las obstrucción intestinales (1); el $43 \%$ al $80 \%$ de los casos son del sigmoide, un $15 \%$ al $42 \%$ del ciego, y tan solo un $2 \%$ al $4 \%$ del colon trasverso $(2,3)$.

Las 2 propiedades fundamentales para la formación del vólvulo son la redundancia y la falta de fijación del segmento colónico; las porciones ascendente y descendente del colon son fijas, pero el colon sigmoide, el ciego y el colon transverso están móviles dentro del peritoneo, sostenidos únicamente por el mesenterio (2).

Se presentan 2 casos de vólvulo del colon transverso espontáneo en un hombre y en una mujer que consultaron por dolor y distensión abdominal. 


\section{REPORTE DE CASO 1}

Hombre de 52 años que consultó al servicio de urgencias por cuadro clínico de 4 días de evolución consistente en dolor abdominal severo, distensión y ausencia de deposiciones, que manejó inicialmente con simeticona y trimebutina sin mejoría. El paciente refirió que el dolor era en epigastrio irradiado hacia la fosa ilíaca derecha y estaba asociado con 2 episodios eméticos de contenido alimenticio y una deposición abundante de características normales. El único antecedente que comentó fue el diagnóstico de síndrome de intestino irritable desde hacía varios años.

En el examen físico, el paciente se encontraba alerta con una deshidratación leve y un abdomen distendido y timpánico, que a la auscultación presentaba disminución de los ruidos intestinales; en la palpación se evidenció distensión abdominal importante con dolor en la palpación generalizada, sin signos claros de irritación peritoneal. El cuadro hemático mostró leucocitosis con neutrofilia, se tomaron pruebas de función renal y parcial de orina que se encontraron normales; en la tomografía de abdomen total con contraste se evidenció una sobre distensión de las asas intestinales gruesas con arremolinamiento de los vasos mesentéricos proyectados sobre el hipocondrio derecho, con presencia de un área de transición en el colon ascendente (figuras 1 y 2 ).

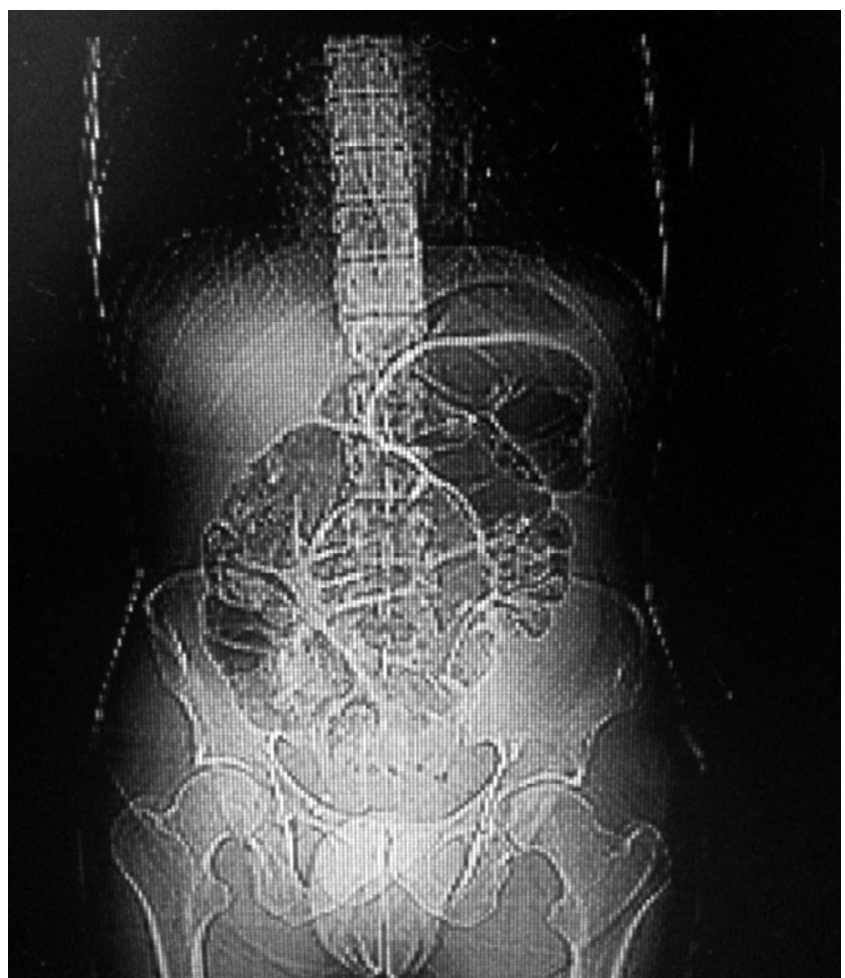

Figura 1. Tomografía de abdomen con contraste.
Debido a los hallazgos, se diagnosticó vólvulo del colon y se decidió realizar devolvulación endoscópica; durante el procedimiento se exploró hasta el ángulo esplénico del colon, y en este nivel se encontró una zona de volvulación no franqueable sin signos de isquemia, por lo que se decidió suspender el procedimiento y trasladar al paciente a sala de cirugía para la realización de una laparotomía. Durante el procedimiento quirúrgico se encontró un dolicocolon, con vólvulo del colon trasverso, con importante dilatación y áreas de isquemia sin perforación; se hizo una liberación del colon ascendente y descendente con pinzamiento, corte y ligadura del mesocolon transverso, y exéresis de la pieza. Luego se realizó una anastomosis laterolateral con sutura mecánica y cierre de boca en 2 planos. Durante el posoperatorio, el paciente presentó una evolución satisfactoria y fue dado de alta en los días siguientes.

\section{REPORTE DE CASO 2}

Paciente femenina de 80 años que consultó al servicio de urgencias por cuadro de 5 días de evolución consistente en múltiples episodios eméticos de contenido alimenticio y dolor abdominal generalizado de moderada intensidad que no respondió a analgésicos y antiespasmódicos. Como antecedentes importantes, la paciente era hipertensa, tenía EPOC y era usuaria de marcapasos por bloqueo AV de tercer grado con antecedentes quirúrgicos de cistopexia e histerectomía.

$\mathrm{Al}$ examen físico, se encontró a la paciente afebril e hipotensa, con abdomen distendido y timpánico, con disminución de los ruidos intestinales en la auscultación y con dolor generalizado, de moderado a severo, en la palpación. En los paraclínicos de ingreso presentó cuadro hemático normal, con BUN de 89 y gases arteriales que mostraban acidosis metabólica severa ( $\mathrm{pH} 7,31)$ con hiperlactatemia (11 mg/ dL); en la radiografía de abdomen se observó distensión de las asas colónicas con niveles hidroaéreos y ausencia de gas distal (figura 3).

Debido a los hallazgos, se diagnosticó vólvulo del colon y se decidió realizar una devolvulación por colonoscopia, que mostró un punto de volvulación en el colon descendente y que se logró revertir y franquear, seguido de un área de dilatación colónica marcada, y un segundo punto de vólvulo a $65 \mathrm{~cm}$ del reborde anal que no se logró franquear (figura 4). Por lo anterior, se decide interconsultar prioritariamente al servicio de cirugía general.

La paciente se lleva a laparotomía luego de aproximadamente 8 horas de su ingreso; durante el procedimiento quirúrgico se encontró peritonitis fecal secundaria de perforación del colon ascendente y vólvulo del colon trasverso, 


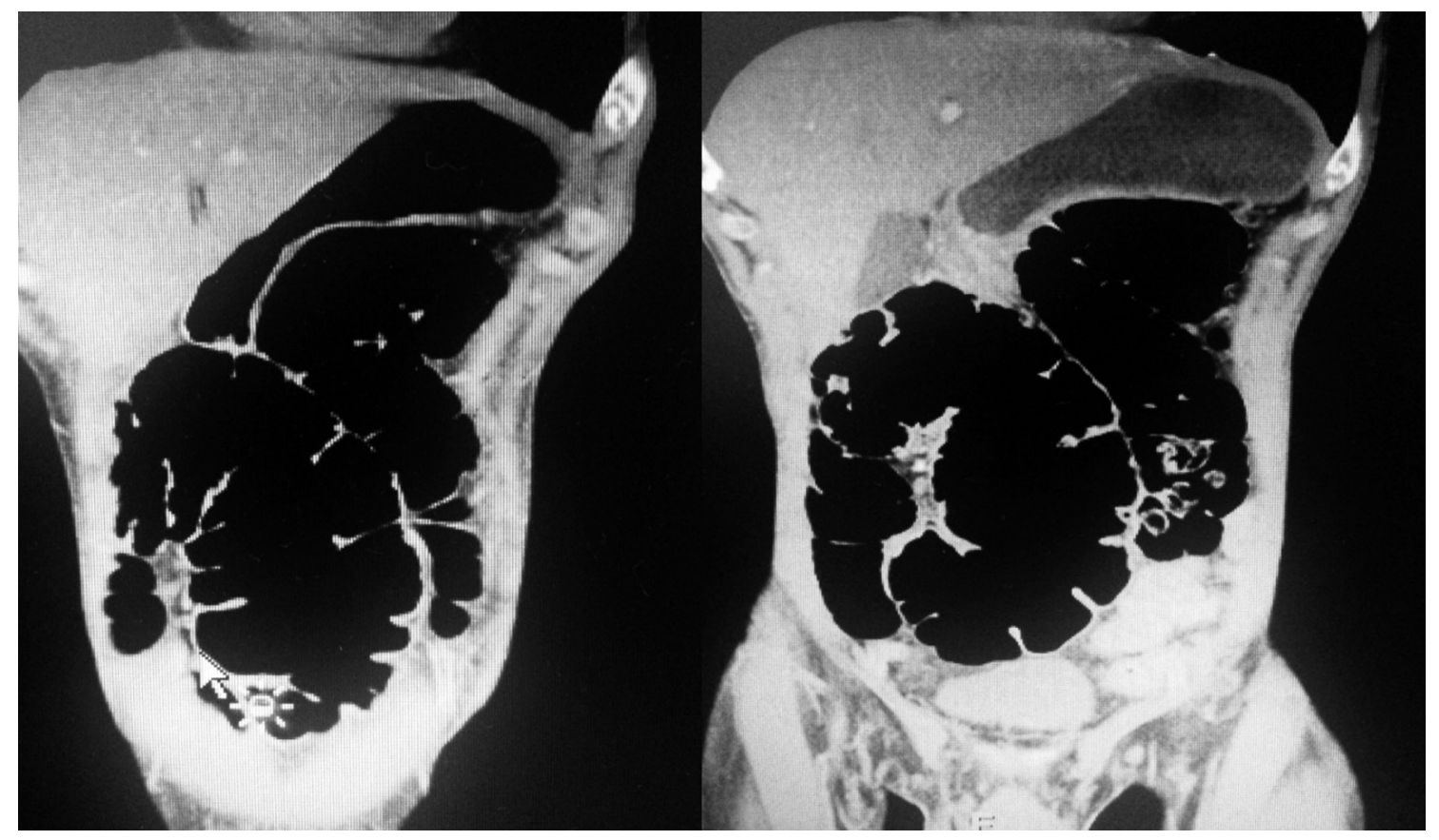

Figura 2. Tomografía de abdomen con contraste.

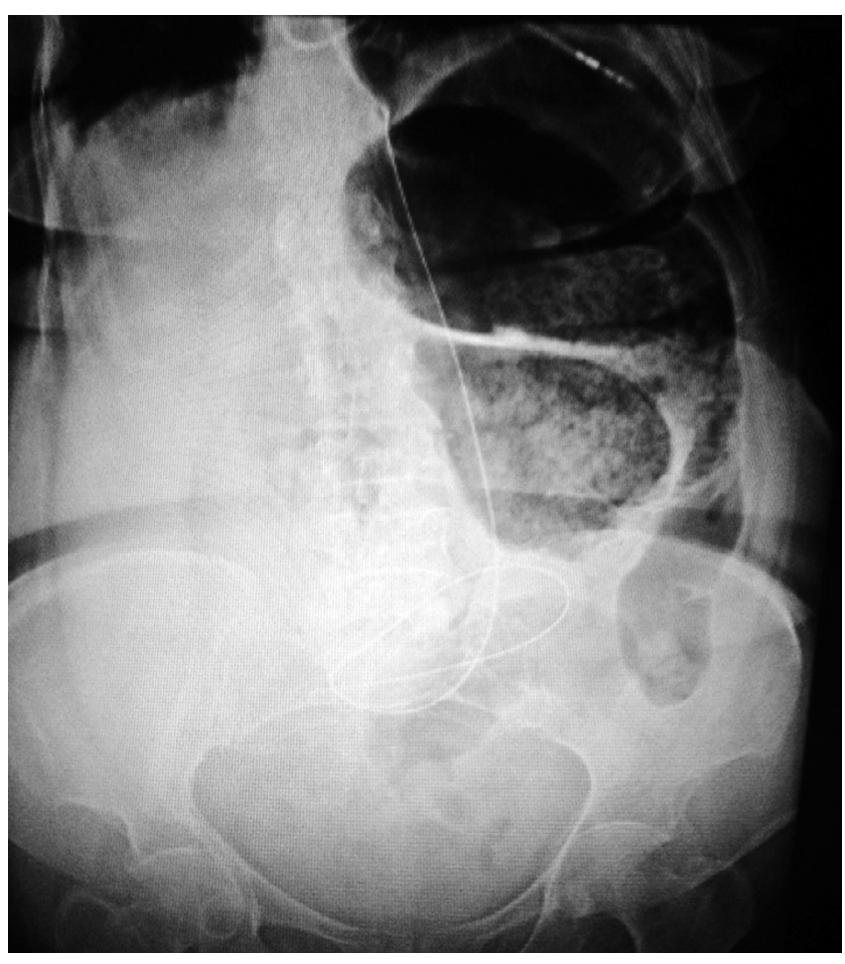

Figura 3. Radiografía de abdomen simple. con marcada friabilidad de los tejidos por hipoperfusión. Se realizó una colectomía subtotal con sangrado en capa secundario de lesión de la arteria esplénica y desgarro hepático que requirió empaquetamiento y laparotomía. En el posoperatorio se trasladó de inmediato a cuidados intensivos con alto requerimiento de soporte vasopresor y transfusión de hemoderivados. A las 24 horas se reinterviene y se evidencia sangrado en capa de lechos quirúrgicos que requirió lavado peritoneal y nuevo empaquetamiento. La paciente falleció luego de 36 horas del posoperatorio.

\section{DISCUSIÓN}

Un vólvulo es una torsión o una rotación sobre el eje axial de una porción del colon con respecto del mesenterio y puede ser de origen primario o secundario (4). A nivel mundial, los vólvulos del colon son la tercera causa de obstrucción intestinal del intestino grueso (2), y el vólvulo del colon transverso solo representa el 3\% de los vólvulos (5). En 1932, el cirujano finlandés Kallio describió el primer caso de vólvulo del transverso (5, 6); luego, en 1981, Anderson hizo una revisión en la literatura mundial de 66 pacientes, $y$ en 1994, Medina y colaboradores documentaron en su trabajo un total de 72 casos (6). 


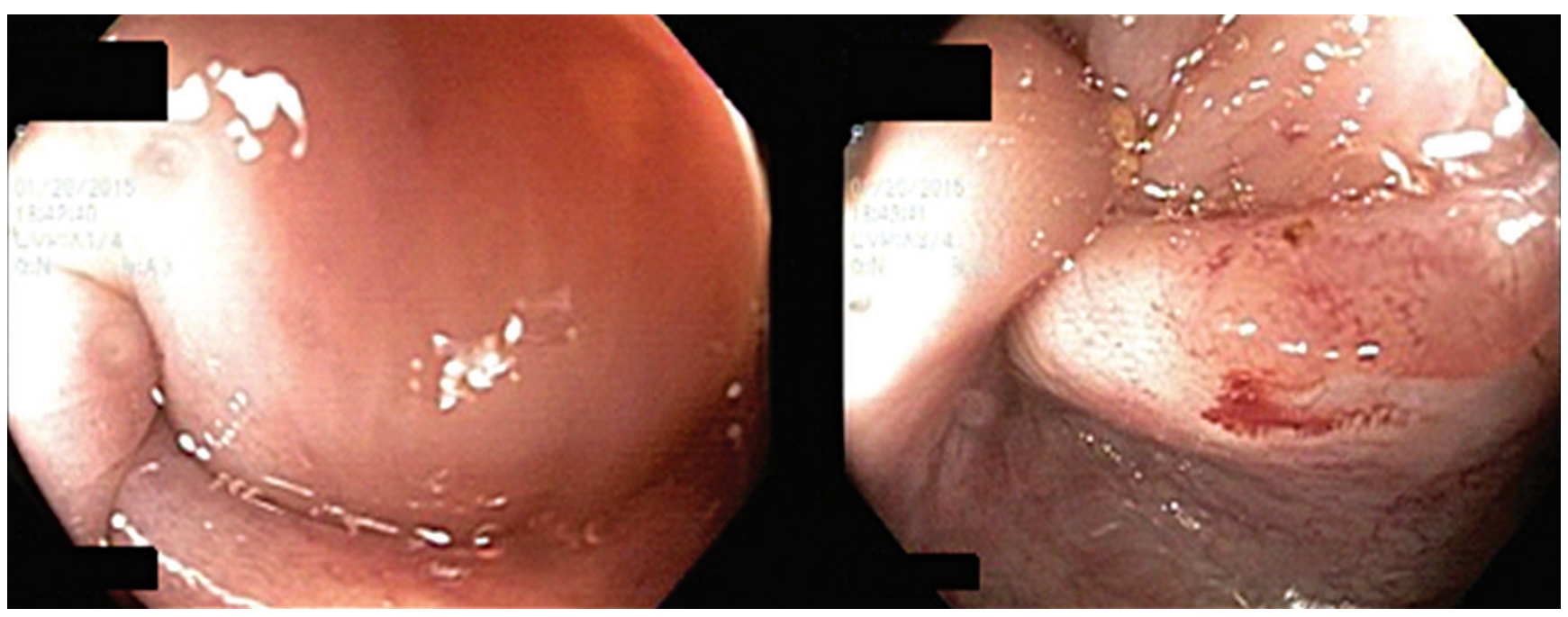

Figura 4. Colonoscopia total: vólvulo a $65 \mathrm{~cm}$ del reborde anal.

Es una entidad que se presenta en 2 picos de edad: pacientes jóvenes en la segunda o tercera década de vida y con un ligero predomino en el género femenino (2), y pacientes ancianos con abundante comorbilidad y con predominio en el género masculino. Los factores que predisponen su desarrollo se clasifican en anatómicos, fisiológicos y mecánicos. Los factores anatómicos más comúnmente asociados son una fijación anormal al mesenterio y errores congénitos en la rotación del intestino medio (7); los fisiológicos incluyen un impedimento distal para la defecación como estreñimiento crónico, elongación y redundancia del colon, y un estrecho punto de fijación al mesenterio $(2,6)$; en los factores mecánicos, el más común es la obstrucción del colon sigmoide que puede ser secundaria de neoplasia, o secuelas de diverticulitis que también pueden desencadenar vólvulos del colon sigmoide (6).

En relación con el cuadro clínico, se han descrito 2 presentaciones: la aguda fulminante o la subaguda progresiva. La primera se caracteriza por náusea y vómito, leucocitosis marcada, dolor abdominal agudo, signos de irritación peritoneal aunque con una distensión abdominal mínima; la presentación subaguda viene acompañada con menos náusea o vómito, leve dolor abdominal, distensión abdominal importante sin signos de irritación peritoneal y conteo de leucocitos normales o levemente aumentados (8).

Con respecto al diagnóstico, generalmente este no se suele hacer de manera preoperatoria, dado que los hallazgos radiográficos son inespecíficos comparados con la imagen típica de grano de café del vólvulo del colon del sigmoide $(4,8)$. Algunos autores mencionan que la presencia de un colon distendido con 2 niveles hidroaéreos en el epigastrio o la presencia de un asa en forma de $U$ invertida en el cuadrante superior izquierdo en la radiografía de abdomen pueden sugerir el diagnóstico, pero su presentación es inconstante $(5,11,12)$. En cuanto a la tomografía, una revisión realizada por Vandendries y colaboradores no reportó características radiológicas propias para el vólvulo del colon transverso (9).

Existe controversia en el manejo del vólvulo intestinal en presencia de un colon viable y sin datos clínicos de peritonismo. Se presenta un acuerdo general en el uso de la colonoscopia descompresiva como tratamiento inicial del vólvulo de colon, independiente del segmento comprometido, con o sin inserción de sonda rectal; la colonoscopia, además de ser una medida terapéutica, permite evaluar el estado de la mucosa colónica y por tanto, la presencia o no de necrosis o signos de isquemia, mostrándose efectiva en más de un $70 \%$ de los pacientes. En caso de fallo en la descompresión endoscópica, lo cual suele ocurrir en la mayoría de los casos de vólvulo de ciego y de colon transverso, será necesario el manejo quirúrgico prioritario $(5,6,13)$.

El manejo quirúrgico es de elección y puede incluir detorsión abierta o laparoscópica, con o sin colopexia, sin embargo, dada la alta tasa de recurrencia, la recomendación es una hemicolectomía extensa derecha o una colectomía del colon transverso (2). Es importante mencionar que en el trabajo publicado por Ballantyne y colaboradores todos los pacientes con vólvulo del colon transverso a los que nada más se les realizó una detorsión con pexia, sin resección intestinal, murieron (10).

\section{CONCLUSIÓN}

El vólvulo del colon transverso es una entidad de presentación infrecuente, con hallazgos radiológicos poco sensibles y cuyo diagnóstico preoperatorio es difícil de realizar con 
certeza. Por este motivo, se debe tener una alta sospecha clínica con el fin de obtener un diagnóstico adecuado y poder ofrecer un manejo oportuno, que en la mayor proporción de pacientes es quirúrgico en forma prioritaria. En cuanto al uso de la colonoscopia, contrario a lo que sucede en el vólvulo del colon sigmoide, no hay evidencia suficiente para recomendar o evitar el procedimiento; sin embargo, sabiendo que su capacidad resolutiva es mínima, y que en caso de conseguirse la devolvulación, la recurrencia es alta, su utilidad debe limitarse al estudio de los diagnósticos diferenciales.

\section{REFERENCIAS}

1. Sana L, Ali G, Kallel H, Amine B, Ahmed S, Ali EM, et al. Spontaneous transverse colon volvulus. Pan Afr Med J. 2013 Jan; 14:160.

2. Gingold D, Murrell Z. Management of Colonic Volvulus. Clin Colon Rectal Surg. 2012;25:236-244.

3. Valsdottir E, Marks JH. Volvulus: Small Bowel and Colon. Clin Colon Rectal Surg. 2008 May;21(2):91-3.

4. Deshmukh SN, Maske AN, Deshpande AP, Shende SP. Transverse colon volvulus with chilaiditis syndrome. Indian J Surg. 2010 Aug;347-9.
5. Walczak DA, Czerwińska M, Fałek W, Trzeciak PW. Volvulus of transverse colon as a rare cause of obstruction - a case report and literature review. Pol Przegl Chir. 2013;605-7.

6. Ramírez-Wiella-Schwuchow G, Villanueva SE, Bolaños BL, García H. Volvulus de colon transverso: reporte de caso. Rev Gastroenterol Mex. 2009;74:35-8.

7. Robin KM Volvulus of the transverse colon. Trop Gastroenterol. 2012;33(3):229-231

8. Sparks DA, Dawood MY, Chase DM, Thomas DJ. Ischemic volvulus of the transverse colon: A case report and review of literature. Cases J. 2008; 1:174.

9. Vandendries C, Jullès MC, Boulay-Coletta I, Loriau J, Zins M. Diagnosis of colonic volvulus: Findings on multidetector CT with three-dimensional reconstructions. Brit J Radiol. 2010;83:983-990.

10. Ballantyne GH, Brandner MD, Beart RWJr, Ilstrup DM. Volvulus of the colon. Incidence and mortality. Ann Surg. 1985;202(1):83-92.

11. Mortensen MJ, Hoffman G. Volvulus of the transverse colon. Postgraduate Med J. 1979; (55), 54-57

12. Newton NE, Reines HD. Transverse Colon Volvulus. Am J Roentgenol. 1977;128:69-72.

13. Mulas C, Bruna M. Management of colonic volvulus. Experience in 75 patients. Rev Esp Enferm Dig. 2010;102 (4):239-248. 